\title{
cAMP Response Element-Binding Protein Is Required for Stress But Not Cocaine-Induced Reinstatement
}

\author{
Arati S. Kreibich and Julie A. Blendy \\ Department of Pharmacology, University of Pennsylvania, Philadelphia, Pennsylvania 19104
}

\begin{abstract}
Reinstatement of previously extinguished conditioned place preference (CPP) is precipitated by stress or drug exposure. Here, we show that acute exposure to forced swim stress (FS), in a context distinct from conditioning, induces reinstatement of cocaine CPP in wild-type mice. This behavior is accompanied by a pattern of phosphorylated cAMP response element-binding protein (pCREB) activation in discrete brain regions that is distinct from the pattern observed after cocaine-induced reinstatement. For example, previous cocaine conditioning increases pCREB levels in the amygdala, and acute exposure to FS, but not to cocaine, further augments these changes. In contrast, previous cocaine conditioning does not alter levels of PCREB in the nucleus accumbens, but acute exposure to FS increases pCREB levels in this region on reinstatement day. Furthermore, to determine whether these alterations of CREB are necessary in FS or cocaine-induced reinstatement, we examined the effect of these stimuli on reinstatement behavior in mice deficient in $\alpha$ and $\Delta$ isoforms of CREB. The CREB ${ }^{\alpha \Delta}$ mutant mice show deficits in FS-induced reinstatement of conditioned place preference. In contrast, they show robust cocaine-induced reinstatement. This deficit in stress but not drug-induced reinstatement indicates a specific requirement for CREB in stress-induced behavioral responses to drugs of abuse.
\end{abstract}

Key words: CREB; FS; reinstatement; RI; conditioned place preference; CPP; cocaine; stress

\section{Introduction}

A key obstacle to successful treatment of drug addiction is relapse, which is frequently precipitated by exposure to stress. Relapse has been modeled in animal studies through reinstatement of responses to drug self-administration or conditioned place preference (CPP) by drug, cue, or stress (de Wit and Stewart, 1981, 1983; Erb et al., 1996; Shalev et al., 2000; Stewart, 2000; McFarland and Kalivas, 2001; Sanchez and Sorg, 2001; Shaham et al., 2003). Stressors such as footshock and restraint, given in the conditioning context, induce reinstatement of previously extinguished response associated with drug reward (Erb et al., 1996; Shaham et al., 2000; Stewart, 2000; Sanchez et al., 2003); however, the efficacy of another common stressor, the forced swim (FS), has never been evaluated in reinstatement behavior. FS has been widely used to detect antidepressant efficacy in animal models (Porsolt et al., 1977; Porsolt, 1979). Animals exposed to FS respond in an active (swimming) or passive (floating) manner to the stress. Importantly, forced swim stress exposure occurs in a context that is distinct from the conditioning environment. To evaluate the significance of this type of stress in reinstatement, we used a single forced swim exposure as a means of reinstating previously extinguished place preference to cocaine in mice.

Reinstatement of conditioned responses to drug reward in-

Received May 4, 2004; revised June 9, 2004; accepted June 14, 2004.

This work was supported by National Institute on Drug Abuse Grants DA-116-49-01A2 (J.A.B.) and T32DA007241-12 (A.S.K.). We thank C. Walters, M. Godfrey, and J. Cleck for their technical assistance and Dr. R. Valentino for critical reading of this manuscript.

Correspondence should be addressed to Julie A. Blendy, 125 John Morgan Building, 3620 Hamilton Walk, Philadelphia, PA 19104-6084.E-mail: blendy@pharm.med.upenn.edu.

D0I:10.1523/JNEUROSCI.1706-04.2004

Copyright $\odot 2004$ Society for Neuroscience $\quad$ 0270-6474/04/246686-07\$15.00/0 volves the mesolimbic dopamine system (Stewart, 1984; Stewart and Vezina, 1988; McFarland and Kalivas, 2001); however, stressors recruit additional brain circuits including the extended amygdala (AMY) [nucleus accumbens (NAc) shell, central amygdala, and the bed nucleus of the stria terminalis (BNST)] (Alheid and Heimer, 1988; de Olmos and Heimer, 1999) to reinstate previously extinguished responses (Shaham et al., 2000, 2003; Erb et al., 2001; Wang et al., 2002; McFarland et al., 2004). Although the brain regions critical in mediating reinstatement have been identified, the molecular underpinnings of this phenomenon remain unknown. The transcription factor cAMP response element-binding protein (CREB) may function at the intersection of both drug reward and stress response. CREB is regulated by both acute and chronic drug treatment. Administration of cocaine has been shown to induce CREB phosphorylation (Kano et al., 1995). Overexpression of the CREB protein in the NAc results in reduced rewarding effects of cocaine (Carlezon et al., 1998). In contrast, decreasing CREB activity by overexpressing a dominant-negative form of CREB (mCREB) results in augmented cocaine reward (Carlezon et al., 1998). Similarly, $\mathrm{CREB}^{\alpha \Delta}$ mutant mice, deficient in $\alpha$ and $\Delta$ isoforms of the CREB protein, demonstrate enhanced responses to the rewarding properties at a low dose of cocaine in a CPP paradigm (Walters and Blendy, 2001). At a higher dose of cocaine, however, this difference is no longer evident (Walters and Blendy, 2001). Although CREB is important in modulating the initial behavioral effects of drugs of abuse, the role of this protein in reinstatement of cocaine reward remains unknown.

Alterations in CREB levels and activation of CREB have been correlated with exposure to stress associated with the FS (BilangBleuel et al., 2002). Overexpressing CREB in the nucleus accum- 


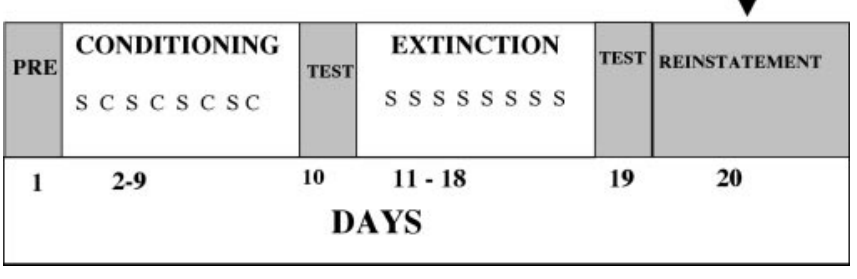

Figure 1. Experimental paradigm for reinstatement. Preconditioning day (PRE): animals are tested for initial bias. Conditioning phase (CONDITIONING): distinct sides of conditioning boxes are paired with either saline (S) or cocaine (C) $(10 \mathrm{mg} / \mathrm{kg}$, i.p.) injections on days $2-9$, with one exposure to box per $24 \mathrm{hr}$. Test is given on day 10 to examine preference. Extinction phase (EXTINCTION): saline injections are given before conditioning on both sides to extinguish preference. Test is given on day 19 to confirm that CPP is extinguished. Arrow represents exposure to either stress or cocaine prime. Stressor consists of a 6 min forced swim test exposure, followed $20 \mathrm{~min}$ later by exposure to the conditioning boxes. Cocaine prime is a cocaine injection (10 $\mathrm{mg} / \mathrm{kg}$, i.p.) given before testing.

bens results in increased immobility in the FS (Pliakas et al., 2001). In contrast, overexpression of mCREB decreases immobility (Pliakas et al., 2001). Convergent findings in our laboratory using $\mathrm{CREB}^{\alpha \Delta}$ mutant mice suggest that CREB mediates specific behavioral responses in the FS stress: mutant mice exposed to the forced swim test exhibit reductions in immobility (Conti et al., 2002).

Because of the central role of CREB in modulating responses to both stress and drugs of abuse, we hypothesized that CREB may be important in mediating stress and drug-induced changes in reinstatement of conditioned place preference. To examine this, we quantified changes in phosphorylated CREB (pCREB) in the brain after reinstatement of conditioned place preference in wild-type animals. To determine whether CREB is necessary for reinstatement to occur, we evaluated the induction of reinstatement in conditioned place preference in $\mathrm{CREB}^{\alpha \Delta}$ mutant mice after FS exposure. Last, changes after cocaine-induced reinstatement were evaluated in wild-type and $\mathrm{CREB}^{\alpha \Delta}$ mutant mice to determine the specificity of the role of CREB in the reinstatement response.

\section{Materials and Methods \\ Animals}

The CREB ${ }^{\alpha \Delta}$ mutant mice and wild-type littermates are bred and maintained in an F1 hybrid background (129SvEvTac/C57BL/6) as described previously (Walters and Blendy, 2001). The parental strains for this hybrid line have been backcrossed with vendor-supplied wild-type strains for several generations. The animals used in this study were obtained by crossing heterozygote $\mathrm{CREB}^{\alpha \Delta}$ 129SvEvTac (N12) to heterozygote $\mathrm{CREB}^{\alpha \Delta}$ C57BL/6 (N10). All mice (2-4 months) were group housed with food and water available ad libitum in an animal care facility on a 12 $\mathrm{hr}$ light/dark cycle (lights on at 7:00 A.M.) in accordance with the University of Pennsylvania Animal Care and Use Committee. All experiments were conducted between 8:00 A.M. and 2:00 P.M.

\section{Behavioral experiments: forced swimming}

Mice were placed for 6 min in plastic cylinders $(23 \mathrm{~cm}$ tall $\times 14 \mathrm{~cm}$ diameter) containing water $\left(23-25^{\circ} \mathrm{C}\right)$ that was $10 \mathrm{~cm}$ deep.

\section{Conditioned place preference}

Place-conditioning boxes consisted of two chambers $(20 \times 20 \times 20 \mathrm{~cm})$, one with stripes on the wall and a metal grid floor and the other with gray walls and plastic flooring. A partition with an opening separated the two chambers in each box, but allowed access to either side of the chamber. This partition was closed off during the pairing days. The conditioned place preference paradigm was performed as follows (Fig. 1).

Preconditioning phase. On day 1, mice were allowed to explore both sides for $900 \mathrm{sec}$, and time spent in each side was recorded. These data were used to separate animals into groups with approximately equal biases for each side.

Conditioning phase. Beginning on day 2, the animals were paired for $8 \mathrm{~d}$, with the saline group receiving injections $(0.9 \%$ sodium chloride) on both sides of the boxes, whereas the drug-paired group received cocaine (10 mg/kg; NIDA Drug Supply, Research Triangle Park, NC) on one side and saline on the other side. Drug-paired sides were randomized among all groups.

Testing phase. On day 9, all of the animals were given a saline injection and allowed to roam freely between the two sides, and time spent on each side was recorded. The data were analyzed and expressed as time spent on the saline-paired side subtracted from time spent on the drug-paired side.

Extinction phase. During extinction training (days 11-19), all animals were given saline injections on both sides of the boxes. On extinction test day (day 20), the time spent on each side was recorded.

Reinstatement phase: stress induced. Twenty-four or $48 \mathrm{hr}$ after extinction test day, mice were placed in a forced swim apparatus for $6 \mathrm{~min}$ and placed back in their home cages. Thirty minutes later, they were tested for place preference, as described previously. Mice in the non-stress (NS) group were left in the home cage until CPP testing.

Reinstatement phase: drug induced. On reinstatement (RI) test day, mice were injected with either saline or cocaine (10 mg/kg, i.p.) immediately before being placed in the testing chamber, as above. Time spent in each side was recorded. Data were analyzed as above.

\section{Statistics}

For all data, statistics were analyzed with ANOVAs using the StatView program. Post hoc tests were conducted with the Bonferroni-Dunn test.

\section{Brain dissections}

Mice were killed immediately after testing on RI day (Fig. 1) by cervical dislocation. The brains were removed and sliced into $1 \mathrm{~mm}$ sections using the brain matrix (Braintree Scientific Co., Braintree, MA). Brain areas of interest [NAc bregma, $+1.54 \mathrm{~mm}$; BNST bregma, $+0.26 \mathrm{~mm}$; AMY bregma, $-1.2 \mathrm{~mm}$; ventral tegmental area (VTA) bregma, -3.88 $\mathrm{mm}$ ] were identified and dissected using the mouse brain atlas (Paxinos and Watson, 1997). Tissues were frozen in liquid nitrogen and stored at $-80^{\circ} \mathrm{C}$.

\section{Western blot analysis}

Tissues were homogenized in $200 \mathrm{ml}$ of ice-cold extraction buffer containing PBS, 1 mm EGTA, 1 mm EDTA, 0.01\% SDS, and 1 mm PMSF. Protein concentrations were determined using a Bradford assay, with bovine serum albumin as the standard. Equivalent amounts of protein $(50 \mu \mathrm{g})$ for each sample were resolved in 7.5\% SDS-PAGE. After electrophoresis, proteins were transferred to polyvinylidene difluoride membranes. Membranes were incubated in PBS with $0.5 \%$ Tween 20 (PBS-T) containing $5 \%$ nonfat milk for $1 \mathrm{hr}$ at room temperature to block nonspecific binding. The blots were reacted with primary antibodies (pCREB or CREB) overnight at $4^{\circ} \mathrm{C}$. After washing in PBS-T, the blots were incubated in secondary antibody in PBS-T for $1 \mathrm{hr}$. Membranes were then washed three times with PBS-T. Immunolabeling was detected by enhanced chemiluminescence (Amersham Biosciences, Piscataway, NJ). The blots were stripped using the standard protocol, washed, and reprobed with the reference antibody ( $\alpha$-tubulin). The primary antibodies used were pCREB, CREB phosphorylated at Ser-133 (1:500; Cell Signaling Technology, Lake Placid, NY), and $\alpha$-tubulin (1:1000; Sigma, St. Louis, MO). The secondary antibodies used were goat anti-rabbit IgGHRP (1:5000) and goat anti-mouse IgG-HRP (1:1000) (Santa Cruz Biotechnology, Santa Cruz, CA). Densitometric analysis was conducted to quantify the immunoreactivity in Western blotting with a scanner and ImageQuant software (Amersham Biosciences). The pCREB bands were detected at $43 \mathrm{kDa} ; \alpha$-tubulin bands were detected at $50 \mathrm{kDa}$. Ratios of pCREB to $\alpha$-tubulin densities were calculated for each sample and analyzed across conditions. 

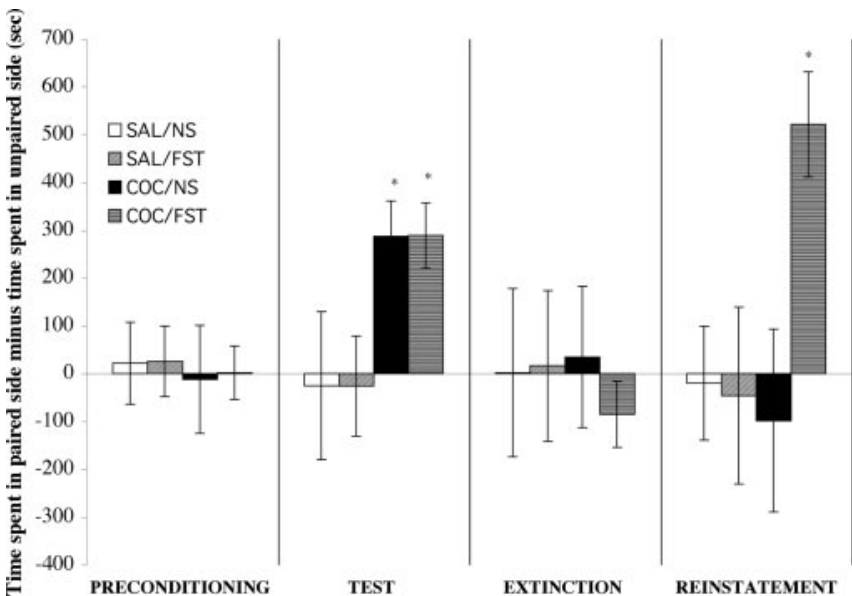

Figure 2. Acute exposure to the forced swim test (FST) precipitates reinstatement of extinguished place preference for cocaine $(10 \mathrm{mg} / \mathrm{kg})$ in wild-type mice. Wild-type mice paired with cocaine (COC/NS, black bar; $\mathrm{COC/FST}$, horizontal stripe) show a significant place preference to the cocaine-paired side on test day. With repeated pairings of saline in both sides, mice previously paired with cocaine do not show place preference on extinction day. When mice are then exposed to a session of acute FST, neither the non-stress groups (SAL/NS, white bars; COC/NS, black bars) nor the saline-paired stressed group (SAL/FST, diagonal stripe) shows significant place preference; however, the mice previously paired with cocaine (COC/FST, horizontal stripe) reveal robust reinstatement of preference after an acute exposure to FST. Data are expressed as mean \pm SEM; five to six mice per group. ${ }^{*} p<0.05$ from corresponding saline group (ANOVA; Bonferroni-Dunn post hoc test).

\section{Results}

Forced swim test induces reinstatement of cocaine place preference in wild-type mice

We evaluated the efficacy of forced swim as a stressor necessary to induce reinstatement in wild-type mice in an F1 hybrid background (129SvEv/C57BL/6). Preconditioning day data revealed no initial bias to either side. On test day, both groups of wild-type mice showed significant place preference to the cocaine-paired side $\left(F_{(3,17)}=3.589 ; p<0.05\right.$ from saline group) (Fig. 2$)$. On extinction test day, none of the groups demonstrated significant place preference. Therefore, reinstatement was tested $24 \mathrm{hr}$ later. Wild-type animals previously demonstrating CPP and exposed to the forced swim stress show a significant preference for the side previously paired with cocaine. In contrast, mice previously injected with cocaine but not subjected to the FS show no significant place preference ( $p>0.05$ from saline group). The FS exposure had no effect on saline-treated animals, which had never developed a preference for one side or another.

\section{pCREB levels increase after FS-induced reinstatement in wild-type mice}

To investigate molecular correlates of the reinstatement behavior, we assessed changes in levels of pCREB in brain areas associated with reward and stress-induced reinstatement in wild-type mice. We found that forced swimming increased pCREB expression in the NAc in both groups, regardless of whether they received saline or cocaine conditioning in $\operatorname{CPP}\left(F_{(3,12)}=14.9 ; p<\right.$ 0.001 from corresponding non-stress group) (Fig. 3A). Conditioning to cocaine alone resulted in increased pCREB levels $10 \mathrm{~d}$ later in the amygdala $\left(F_{(3,12)}=32.4 ; p<0.001\right.$ from the corresponding NS group) (Fig. $3 B$ ). Of note, this occurs $10 \mathrm{~d}$ after the cocaine-conditioning procedure. A single exposure to the FS further augmented pCREB levels in the amygdala in mice that were previously conditioned to cocaine $\left(F_{(3,12)}=32.4 ; p<0.001\right.$ from corresponding cocaine-conditioned, non-stressed group). There
A) Nucleus Accumbens (NAc)
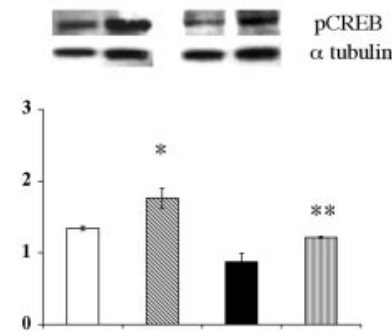

CPP SAL SAL COC COC

RI NS FST NS FST

C) Ventral Tegmental Area (VTA)

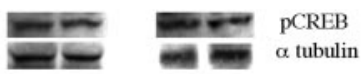

B) Amygdala (AMY)

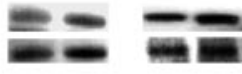

pCREB
$\alpha$ tubulin

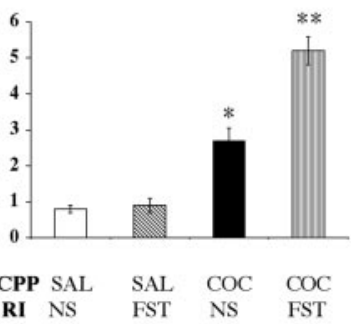

D) Bed Nucleus of the Stria Terminalis (BNST)

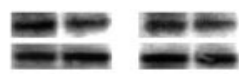

pCRFB

$\alpha$ tubulin
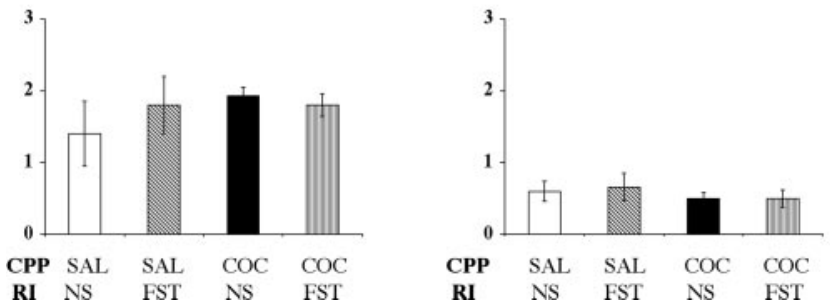

Figure 3. Increases in pCREB after FST-induced reinstatement in wild-type mice. Western immunoblots of pCREB change after FST-induced reinstatement. Wild-type mice were paired with saline (SAL) or cocaine (COC). Of those, some were exposed to swim stress on RI day (SAL/FST or COC/FST); the rest were not stressed (SAL/NS or COC/NS). A, Acute exposure to forced swimming significantly increases pCREB expression in the NAc after both saline (SAL/ FST) and cocaine (COC/FST) conditioning when compared with the corresponding non-stress groups (SAL/NS or COC/NS). B, Cocaine conditioning increases pCREB expression in the amygdala (COC/NS) when compared with all other groups. Acute exposure to forced swimming (COC/FST) further increases pCREB levels when compared with the NS group. C, There were no changes in pCREB expression after forced swimming or cocaine conditioning in the VTA or the BNST $(D)$. Data are expressed as mean \pm SEM; four to six mice per group. ${ }^{*} p<0.05$ from SAL/NS group; ${ }^{* *} p<0.05$ from COC/NS group (ANOVA; Bonferroni-Dunn post hoc test).

were no significant changes in pCREB levels in the VTA or the BNST (Fig. 3C,D).

\section{CREB mutant mice do not show FS-induced reinstatement of} place preference to cocaine

To determine whether changes in PCREB are necessary for stressinduced reinstatement, we tested the effect of FS on reinstatement to place preference in $\mathrm{CREB}^{\alpha \Delta}$ mice. Both wild-type and mutant mice injected with cocaine $(10 \mathrm{mg} / \mathrm{kg})$ showed a similar preference for the drug-paired side on test day $\left(F_{(7,65)}=5.785\right.$; $p<0.001$ from corresponding saline group) (Fig. 4). These data are comparable with previous experiments (Walters and Blendy, 2001). This preference was no longer evident after extinction in either wild-type or mutant mice $\left(F_{(7,65)}=0.429 ; p>0.5\right.$ from all other groups). As demonstrated previously, the wild-type animals showed FS-induced reinstatement $\left(F_{(7,65)}=3.410 ; p<\right.$ 0.001 from wild-type saline group). In contrast, $\mathrm{CREB}^{\alpha \Delta}$ mutant animals do not show reinstatement after FS exposure $\left(F_{(7,65)}=\right.$ 3.410; $p>0.5$ from all other groups) (Fig. 4).

Cocaine priming induces reinstatement of cocaine place preference in wild-type and CREB ${ }^{\alpha \Delta}$ mutant mice

To further assess whether the deficit in reinstatement in $\mathrm{CREB}^{\alpha \Delta}$ mutant mice is specific to stress, we evaluated whether a cocaine 


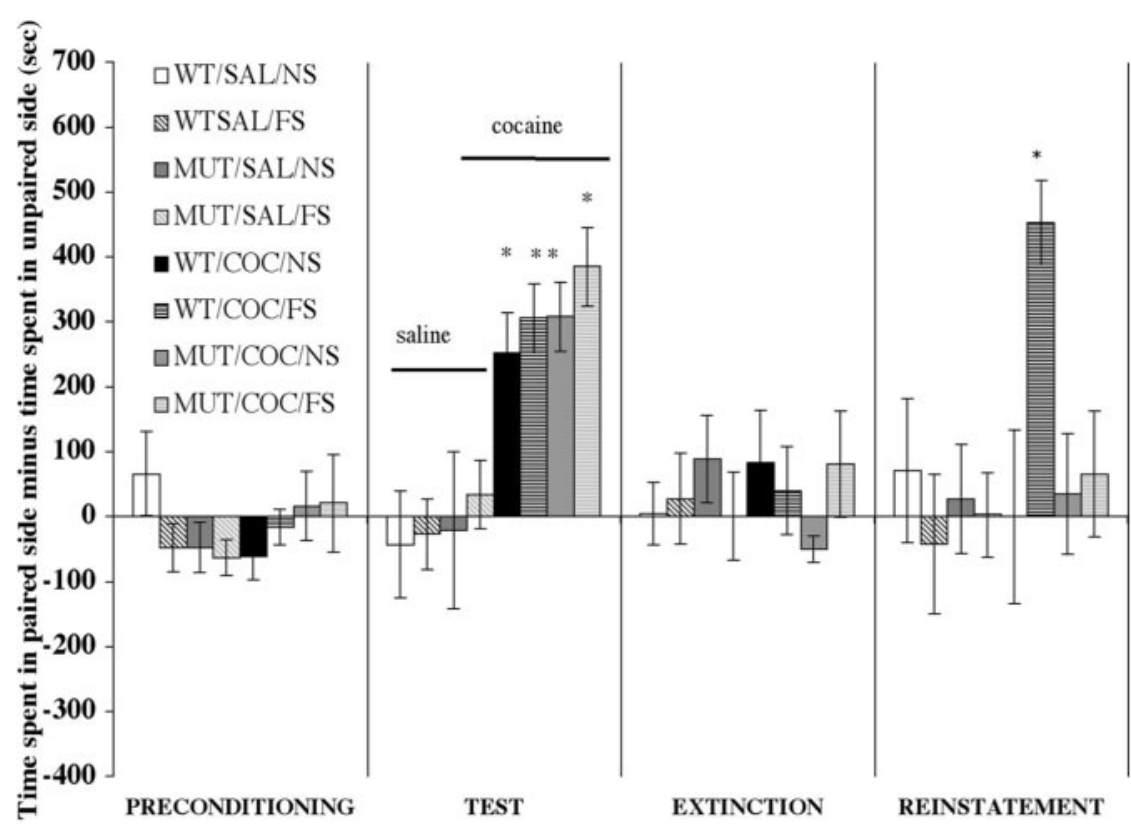

Figure 4. CREB mutant mice do not show FST-induced reinstatement of place preference to cocaine $(10 \mathrm{mg} / \mathrm{kg})$. Both wild-type and mutant mice paired with cocaine (WT/COC/NS, WT/COC/FST, MUT/COC/NS, MUT/COC/FST) show a significant place preference to the cocaine-paired side on test day. With repeated pairings of saline in both sides, mice previously paired with cocaine do not show place preference on extinction day. Although wild-type mice reveal reinstatement of preference after a single exposure to FST (WT/COC/FST), the CREB mutant mice do not show reinstatement (MUT/COC/FST). Data are expressed as mean \pm SEM; $10-12$ mice per group. ${ }^{*} p<0.05$ from corresponding saline group (ANOVA; Bonferroni-Dunn post hoc test).

priming injection would induce reinstatement of place preference in both wild-type and $\mathrm{CREB}^{\alpha \Delta}$ mice. As shown previously, both wild-type and mutant animals showed place preference to cocaine $\left(F_{(7,37)}=6.243 ; p<0.001\right.$ from corresponding saline group) and an extinction of this preference after saline pairings $\left(F_{(7,37)}=0.168 ; p>0.5\right.$ from saline group) (Fig. 5). In contrast to FS-induced reinstatement, both wild-type and $\mathrm{CREB}^{\alpha \Delta}$ mutant mice showed similar reinstatement of place preference elicited via cocaine prime injection $\left(F_{(7,37)}=3.165 ; p<0.01\right.$ from all corresponding groups) (Fig. 5).

\section{pCREB increases after cocaine-induced reinstatement in wild-type mice}

To evaluate whether the changes in PCREB are specific to stressinduced reinstatement, we investigated changes in pCREB after cocaine-induced reinstatement. Cocaine injections on reinstatement day had no effect on pCREB levels in the NAc (Fig. 6A). As seen previously, cocaine conditioning itself increased pCREB levels in the amygdala $\left(F_{(3,12)}=3.7 ; p<0.05\right.$ from corresponding saline group); however, exposure to an acute injection of cocaine on reinstatement day did not further increase pCREB levels in the amygdala (Fig. 6B). Cocaine prime injection increased pCREB levels only in the VTA in animals previously conditioned to cocaine (Fig. $6 C)\left(F_{(3,12)}=4.2 ; p<0.05\right.$ from corresponding cocaine-conditioned, saline-injected group). We found no changes in pCREB expression after cocaine conditioning or cocaine injections on RI day in the NAc or the BNST (Fig. 6A,D).

\section{Discussion}

Exposure to stress may be a determining factor in the vulnerability to substance abuse. Specifically, stress has been shown to increase the acquisition of drug self-administration in both rodent and nonhuman primates (Kraemer and McKinney, 1985; Higley et al., 1991; Piazza and Le Moal, 1998; Fahlke et al., 2000). In general, to elicit stress-induced reinstatement of place preference, the stressor must be given in the same context as the drug conditioning. For example, both footshock and restraint stress given within the self-administration chambers reinstate heroin-seeking behavior (Shalev et al., 2000). In contrast, neither footshock nor restraint stress given outside the conditioning context has been shown to reinstate drug seeking (Shalev et al., 2000). External stressors outside of the drug-taking environment do induce relapse in humans (Shiffman, 1982; Sinha et al., 1999, 2000). Therefore, similar evaluations of reinstatement would be useful to examine in animal models of relapse.

Forced swim stress is a common behavioral paradigm used to evaluate antidepressant efficacy. In addition to the assessment of physiological changes associated with stress, FS allows for the observation and quantification of behavioral responses during the stress exposure. In the present studies, we have shown that exposure to FS reinstates cocaine place preference in mice. Forced swimming was performed in a cylinder of water in an area separate from the conditioned place preference chambers, demonstrating that exposure to a stress in an environment distinct from conditioning can induce reinstatement of place preference. More recently, deprivation of food has been used to elicit reinstatement (Shalev et al., 2001; Highfield et al., 2002). It remains the only other example of stress exposure in a context different from conditioning that elicits reinstatement; however, deprivation of food may be characterized as an "internal" stressor, wherein changes in the state of the animal occur over the long-term period of 1 or more days. In contrast, exposure to FS is an acute, discrete "external" stressor. Thus, this is the first demonstration of an external stressor that elicits reinstatement when given in a different context than the conditioning paradigm.

Although substantial evidence points to a relationship between stress and drug addiction, the molecular mechanisms underlying this link have yet to be elucidated. The transcription factor CREB is considered central in transducing responses to drugs of abuse as well as to stressors. To investigate the role of CREB in stress and drug-induced changes in response to cocaine, we examined pCREB protein levels after FS and cocaine-induced reinstatement in the NAc, VTA, AMY, and BNST. These brain regions are implicated in mediating reinstatement to drugs as well as to stress (Erb and Stewart, 1999; Erb et al., 2000, 2001; McFarland and Kalivas, 2001; McFarland et al., 2004). In addition, the NAc and the VTA are thought to be part of the final common motor pathway by which all priming stimuli reinstate behavior. Inactivation of both regions abolishes footshock and drug-induced reinstatement (McFarland and Kalivas, 2001; McFarland et al., 2004). Our data demonstrate that pCREB levels are increased in the nucleus accumbens on reinstatement day after an exposure to forced swim test. This increase was seen regardless of whether animals were treated with saline or cocaine in the conditioning paradigm, indicating that activation of CREB is caused 
primarily by exposure to the FS. In contrast, pCREB levels did not increase after cocaine-induced RI in the NAc, suggesting that activation of pCREB in the NAc is required for the FS to elicit reinstatement in this paradigm.

Acute exposure to the FS has also been shown to change excitatory synaptic plasticity in the VTA (Saal et al., 2003). Moreover, this change is similar to that observed after exposure to drugs of abuse (Saal et al., 2003); however, we found no changes in pCREB levels in the VTA after cocaine conditioning or FS exposure. This is in contrast to the increase in PCREB observed in the VTA after an acute injection of cocaine on reinstatement day. Because the VTA mediates the initial response to drugs of abuse, the increase in pCREB after the cocaine prime injection may reflect acute drug-dependent changes in the VTA. The pattern of pCREB changes that emerges after cocaine-induced reinstatement is distinct from that observed after FS-induced RI, indicating that distinct molecular mechanisms in discrete brain regions may be differentially involved in stress- versus druginduced reinstatement.

The amygdala and BNST are additional critical neural substrates involved in stress-induced reinstatement. Inactivation of the central amygdala or the BNST attenuates stress-induced reinstatement (Erb and Stewart, 1999; Erb et al., 2000, 2001; Wang et al., 2001, 2002). Although levels of pCREB are not altered in the BNST by exposure to the FS, alterations of pCREB in the amygdala are significantly affected by this reinstatement stimulus. Moreover, previous conditioning to cocaine itself increases pCREB levels in the amygdala of wild-type mice. This suggests that long-lasting changes in the amygdala may serve to "sensitize" the system for subsequent reinstatement. Of interest, although exposure to the FS augments this increase in pCREB, acute cocaine given on reinstatement day does not. Although both the amygdala and the BNST are implicated in mediating other forms of stress-induced reinstatement, CREB regulation appears to be critical to the amygdala after FS-induced reinstatement.

Analysis of pCREB levels shows distinct patterns of activation depending on the stimulus used to induce reinstatement. The nucleus accumbens and the amygdala are differentially activated after FS and conditioning to cocaine. These regions, along with the BNST, comprise the "extended amygdala" region (Alheid and Heimer, 1988; de Olmos and Heimer, 1999). As such, there are extensive reciprocal connections between them (Cassell et al., 1999). The pattern of changes in pCREB observed after acute stress exposure as well as conditioning may reveal the convergence of connections in these brain regions, and upregulation of CREB in both brain regions may be necessary for FS-induced reinstatement. In contrast, the pCREB activation is seen in the VTA only after an acute cocaine injection on RI day. Together, these data show that discrete brain regions may be differentially involved in a specific pattern of neuronal activation in distinct types of reinstatement.

To determine whether CREB is necessary for stress- or druginduced reinstatement, we evaluated these behaviors in mice de- ficient in the CREB protein: $\mathrm{CREB}^{\alpha \Delta}$ mice. We have shown that mutant mice deficient in CREB do not show FS-induced reinstatement. In contrast, we found that both wild-type and mutant mice show robust reinstatement to a cocaine priming injection. These data indicate that the behavioral deficit in reinstatement in the $\mathrm{CREB}^{\alpha \Delta}$ mice is specific to stress and not a global deficit in expressing a reinstatement response.

Augmentation of the pCREB response in the amygdala occurs after FS-induced reinstatement and in the NAc after FS exposure in wild-type mice. In addition, we also observe deficits in FSinduced reinstatement in $\mathrm{CREB}^{\alpha \Delta}$ mice. Thus, the behavioral deficit observed is consistent with the increases in PCREB after FS-induced reinstatement. Hence, dysregulation of intracellular signaling in the amygdala may interfere with stress-induced reinstatement processes in the CREB-deficient mice. Taken together, these data indicate that CREB in the amygdala and the NAc is critical to elicit stress-induced reinstatement; however, it appears that pCREB changes in the VTA after cocaine injection are not essential in mediating behavioral response to cocaine-induced reinstatement.

This deficit in stress but not drug prime-induced reinstatement for extinguished place preference indicates a specific requirement for CREB in stress-induced behavioral responses to drugs of abuse. The mechanisms underlying this response may be twofold. Inactivation of the CRF input from the central nucleus of the amygdala to the BNST abolishes stress-induced reinstatement in conjunction with corticotropin releasing factor receptor 1 (CRFR1) receptor blockade in the BNST (Erb and Stewart, 1999; Erb et al., 2001). Increases in levels of pCREB indicate the involvement of the amygdala specifically in acute FS-induced reinstatement of extinguished place preference in wild-type mice. CREB acts on CRE elements present in the promoter of various genes, including that of the corticotropin-releasing factor gene (Hatalski and Baram, 1997). Therefore, in wild-type mice, CREB 
A) Nucleus Accumbens (NAc)

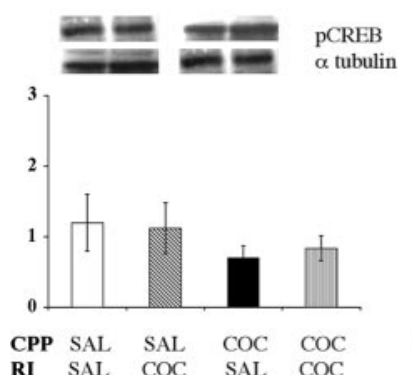

C) Ventral Tegmental Area (VTA)
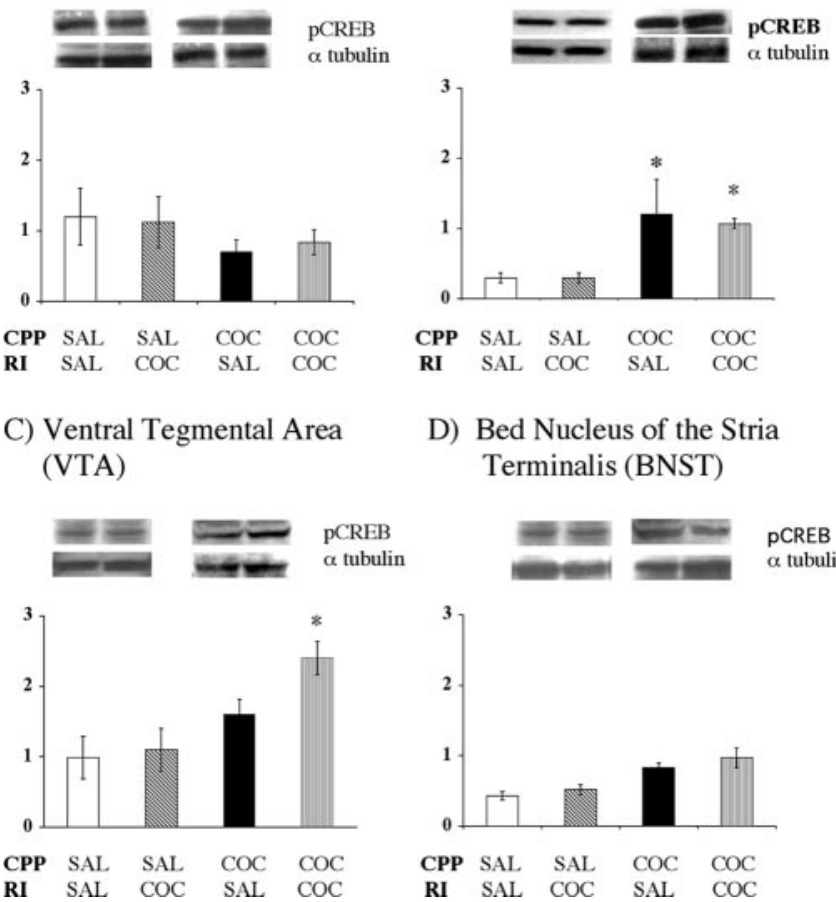

D) Bed Nucleus of the Stria Terminalis (BNST)

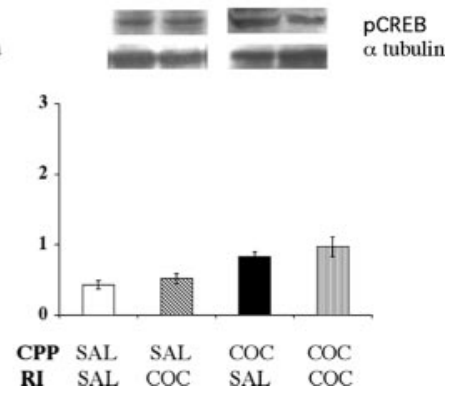

Figure 6. Increases in pCREB after cocaine-induced reinstatement in wild-type mice. Western immunoblots of $p C R E B$ changes after cocaine prime-induced reinstatement. Wild-type mice were paired with saline (SAL) or cocaine $(C O C)$. One group in each condition was exposed to cocaine prime injection before reinstatement testing $(\mathrm{SAL} / \mathrm{COC}$ or $\mathrm{COC} / \mathrm{COC})$; the other group was injected with saline (SAL/SAL or COC/SAL). A, Priming injection of cocaine prime does not increase pCREB expression in the NAc. B, Cocaine conditioning (COC/COC and COC/SAL) significantly increases pCREB expression when compared with saline conditioning (SAL/COC and SAL/ $S A L)$ in the amygdala; however, there is no effect of cocaine prime injection on pCREB levels in the amygdala. C, Injection of the cocaine prime in cocaine-conditioned animals significantly increases pCREB expression in the VTA when compared with corresponding saline group (COC) $C O C$ vs SAL/COC). D, There are no significant differences in pCREB expression in the BNST across any of the groups. Data are expressed as mean \pm SEM; four to six mice per group. ${ }^{*} p<0.05$ from corresponding saline-conditioned group (ANOVA; Bonferroni-Dunn post hoc test).

may act presynaptically to increase CRF production or release, leading to the precipitation of stress-induced reinstatement. $\mathrm{CREB}^{\alpha \Delta}$ mice show a global deficit in CREB, including in the amygdala. Decreases in CREB throughout development and after stimulation may lead to a decrease in baseline limbic production or stress-induced release of CRF. In addition, activation of CRFR1 is necessary to establish stress-induced reinstatement (Erb et al., 1999). CRFR1 is also coupled to the cAMP pathway and thus to CREB (Rossant et al., 1999; Bayatti et al., 2003). Therefore, deficits in CREB may uncouple CRFR1 receptors, leading to an attenuation of signal after stimulation. These baseline or stress-induced changes in CRF or CRFR1 may thus block stress-induced reinstatement response.

In conclusion, we have shown that exposure to FS, a discrete behavioral stressor, in a distinct context can precipitate reinstatement of previously extinguished place preference. Furthermore, changes in CREB in the limbic system (NAc and AMY) may be important for stress-induced reinstatement; however, changes in CREB in the VTA, although critical for the initial reinforcing effects of drugs of abuse, may be less important for drug-induced reinstatement. Global CREB deficiency yields deficits in forced swim-induced but not cocaine-induced reinstatement of place preference. Therefore, pharmacotherapeutic approaches that focus on regulation of CREB and its target genes such as CRF in the limbic system hence may be effective tools in preventing relapse.

\section{References}

Alheid GF, Heimer L (1988) New perspectives in basal forebrain organization of special relevance for neuropsychiatric disorders: the striatopallidal, amygdaloid, and corticopetal components of substantia innominata. Neuroscience 27:1-39.

Bayatti N, Zschocke J, Behl C (2003) Brain region-specific neuroprotective action and signaling of corticotropin-releasing hormone in primary neurons. Endocrinology 144:4051-4060.

Bilang-Bleuel A, Rech J, De Carli S, Holsboer F, Reul JM (2002) Forced swimming evokes a biphasic response in CREB phosphorylation in extrahypothalamic limbic and neocortical brain structures in the rat. Eur J Neurosci 15:1048-1060.

Carlezon Jr AW, Thome J, Olson VG, Lane-Ladd SB, Brodkin ES, Hiroi N, Duman RS, Neve RL, Nestler EJ (1998) Regulation of cocaine reward by CREB. Science 282:2272-2275.

Cassell MD, Freedman LJ, Shi C (1999) The intrinsic organization of the central extended amygdala. Ann NY Acad Sci 877:217-241.

Conti AC, Cryan JF, Dalvi A, Lucki I, Blendy JA (2002) cAMP response element-binding protein is essential for the upregulation of brain-derived neurotrophic factor transcription, but not the behavioral or endocrine responses to antidepressant drugs. J Neurosci 22:3262-3268.

de Olmos JS, Heimer L (1999) The concepts of the ventral striatopallidal system and extended amygdala. Ann NY Acad Sci 877:1-32.

de Wit H, Stewart J (1981) Reinstatement of cocaine-reinforced responding in the rat. Psychopharmacology (Berl) 75:134-143.

de Wit H, Stewart J (1983) Drug reinstatement of heroin-reinforced responding in the rat. Psychopharmacology (Berl) 79:29-31.

Erb S, Stewart J (1999) A role for the bed nucleus of the stria terminalis, but not the amygdala, in the effects of corticotropin-releasing factor on stressinduced reinstatement of cocaine seeking. J Neurosci 19:RC35(1-6).

Erb S, Shaham Y, Stewart J (1996) Stress reinstates cocaine-seeking behavior after prolonged extinction and a drug-free period. Psychopharmacology (Berl) 128:408-412.

Erb S, Hitchcott PK, Rajabi H, Mueller D, Shaham Y, Stewart J (2000) Alpha-2 adrenergic receptor agonists block stress-induced reinstatement of cocaine seeking. Neuropsychopharmacology 23:138-150.

Erb S, Salmaso N, Rodaros D, Stewart J (2001) A role for the CRFcontaining pathway from central nucleus of the amygdala to bed nucleus of the stria terminalis in the stress-induced reinstatement of cocaine seeking in rats. Psychopharmacology (Berl) 158:360-365.

Fahlke C, Lorenz JG, Long J, Champoux M, Suomi SJ, Higley JD (2000) Rearing experiences and stress-induced plasma cortisol as early risk factors for excessive alcohol consumption in nonhuman primates. Alcohol Clin Exp Res 24:644-650.

Franklin KBJ, Paxinos G (1997) The mouse brain stereotaxic coordinates. San Diego: Academic.

Hatalski CG, Baram TZ (1997) Stress-induced transcriptional regulation in the developing rat brain involves increased cyclic adenosine $3^{\prime}, 5^{\prime}$ monophosphate-regulatory element binding activity. Mol Endocrinol 11:2016-2024

Highfield DA, Mead AN, Grimm JW, Rocha BA, Shaham Y (2002) Reinstatement of cocaine seeking in 129X1/SvJ mice: effects of cocaine priming, cocaine cues and food deprivation. Psychopharmacology (Berl) 161:417-424.

Higley JD, Hasert MF, Suomi SJ, Linnoila M (1991) Nonhuman primate model of alcohol abuse: effects of early experience, personality, and stress on alcohol consumption. Proc Natl Acad Sci USA 88:7261-7265.

Kano T, Suzuki Y, Shibuya M, Kiuchi K, Hagiwara M (1995) Cocaineinduced CREB phosphorylation and c-Fos expression are suppressed in Parkinsonism model mice. NeuroReport 6:2197-2200.

Kraemer GW, McKinney WT (1985) Social separation increases alcohol consumption in rhesus monkeys. Psychopharmacology (Berl) 86:182-189.

McFarland K, Kalivas PW (2001) The circuitry mediating cocaine-induced reinstatement of drug-seeking behavior. J Neurosci 21:8655-8663.

McFarland K, Davidge SB, Lapish CC, Kalivas PW (2004) Limbic and motor circuitry underlying footshock-induced reinstatement of cocaine-seeking behavior. J Neurosci 24:1551-1560. 
Piazza PV, Le Moal M (1998) The role of stress in drug self-administration. Trends Pharmacol Sci 19:67-74.

Pliakas AM, Carlson RR, Neve RL, Konradi C, Nestler EJ, Carlezon Jr WA (2001) Altered responsiveness to cocaine and increased immobility in the forced swim test associated with elevated cAMP response elementbinding protein expression in nucleus accumbens. J Neurosci 21:7397-7403.

Porsolt RD (1979) Animal model of depression. Biomedicine 30:139-140.

Porsolt RD, Bertin A, Jalfre M (1977) Behavioral despair in mice: a primary screening test for antidepressants. Arch Int Pharmacodyn Ther 229:327-336.

Rossant CJ, Pinnock RD, Hughes J, Hall MD, McNulty S (1999) Corticotropin-releasing factor type 1 and type 2 alpha receptors regulate phosphorylation of calcium/cyclic adenosine $3^{\prime}, 5^{\prime}$ monophosphate response element-binding protein and activation of p42/p44 mitogenactivated protein kinase. Endocrinology 140:1525-1536.

Saal D, Dong Y, Bonci A, Malenka RC (2003) Drugs of abuse and stress trigger a common synaptic adaptation in dopamine neurons. Neuron 37:577-582.

Sanchez CJ, Sorg BA (2001) Conditioned fear stimuli reinstate cocaineinduced conditioned place preference. Brain Res 908:86-92.

Sanchez CJ, Bailie TM, Wu WR, Li N, Sorg BA (2003) Manipulation of dopamine d1-like receptor activation in the rat medial prefrontal cortex alters stress- and cocaine-induced reinstatement of conditioned place preference behavior. Neuroscience 119:497-505.

Shaham Y, Erb S, Stewart J (2000) Stress-induced relapse to heroin and cocaine seeking in rats: a review. Brain Res Brain Res Rev 33:13-33.

Shaham Y, Shalev U, Lu L, De Wit H, Stewart J (2003) The reinstatement model of drug relapse: history, methodology and major findings. Psychopharmacology (Berl) 168:3-20.

Shalev U, Highfield D, Yap J, Shaham Y (2000) Stress and relapse to drug seeking in rats: studies on the generality of the effect. Psychopharmacology (Berl) 150:337-346.

Shalev U, Yap J, Shaham Y (2001) Leptin attenuates acute food deprivationinduced relapse to heroin seeking. J Neurosci 21:RC129(1-5).

Shiffman S (1982) Relapse following smoking cessation: a situational analysis. J Consult Clin Psychol 50:71-86.

Sinha R, Catapano D, O’Malley S (1999) Stress-induced craving and stress response in cocaine dependent individuals. Psychopharmacology (Berl) 142:343-351.

Sinha R, Fuse T, Aubin LR, O'Malley SS (2000) Psychological stress, drugrelated cues and cocaine craving. Psychopharmacology (Berl) 152:140-148.

Stewart J (1984) Reinstatement of heroin and cocaine self-administration behavior in the rat by intracerebral application of morphine in the ventral tegmental area. Pharmacol Biochem Behav 20:917-923.

Stewart J (2000) Pathways to relapse: the neurobiology of drug- and stressinduced relapse to drug-taking. J Psychiatry Neurosci 25:125-136.

Stewart J, Vezina P (1988) A comparison of the effects of intra-accumbens injections of amphetamine and morphine on reinstatement of heroin intravenous self-administration behavior. Brain Res 457:287-294.

Walters CL, Blendy JA (2001) Different requirements for cAMP response element-binding protein in positive and negative reinforcing properties of drugs of abuse. J Neurosci 21:9438-9444.

Wang B, Luo F, Ge XC, Fu AH, Han JS (2002) Effects of lesions of various brain areas on drug priming or footshock-induced reactivation of extinguished conditioned place preference. Brain Res 950:1-9.

Wang X, Cen X, Lu L (2001) Noradrenaline in the bed nucleus of the stria terminalis is critical for stress-induced reactivation of morphineconditioned place preference in rats. Eur J Pharmacol 432:153-161. 